\title{
The elementary-equivalence classes of clopen algebras of $P$-spaces
}

\author{
by \\ Brian Wynne (Great Barrington, MA)
}

\begin{abstract}
Two Boolean algebras are elementarily equivalent if and only if they satisfy the same first-order statements in the language of Boolean algebras. We prove that every Boolean algebra is elementarily equivalent to the algebra of clopen subsets of a normal $P$-space.
\end{abstract}

1. Introduction. Let $X$ be a topological space and $\operatorname{Clop}(X)$ the set of all clopen subsets of $X$. $\operatorname{Clop}(X)$ is a Boolean algebra under the operations of set-theoretic union, intersection, and complementation. Conversely, by a famous theorem of M. Stone [5], every Boolean algebra is isomorphic to the algebra of clopen subsets of a Boolean space, i.e., a space that is compact, Hausdorff, and zero-dimensional. Recall that a $P$-space is a completely regular space in which every $G_{\delta}$-set is open. The clopen algebras of $P$-spaces are $\sigma$-complete and hence are less diverse than those of Boolean spaces. However, if we require only classification up to elementary equivalence, a relation from mathematical logic weaker than isomorphism, then an analogue of Stone's result is available. Specifically, we intend to prove

THEOREM 1.1. Every Boolean algebra is elementarily equivalent to the algebra of clopen subsets of a normal P-space.

In [7] Theorem 1.1 plays an essential role in proofs of model-theoretic properties of certain lattice-ordered groups of continuous functions.

2. The Tarski invariants. We assume familiarity with the basic notions of Boolean algebra; for all undefined terms we refer the reader to Chapter 1 of [4]. We consider Boolean algebras as structures for the first-order language $\{+, \cdot,-, 0,1\}$, where + is interpreted as join, · as meet, - as complement, and 0 and 1 as the bottom and top elements, respectively, in the algebra.

2000 Mathematics Subject Classification: Primary 54G10, 06E99; Secondary 03C07. Key words and phrases: $P$-space, Boolean algebra, elementary equivalence. 
In [6] Tarski associates with each Boolean algebra a triple of numerical invariants that completely determines its elementary-equivalence class. These triples encode information about certain ideals of the algebra. Let $A$ be a Boolean algebra and $I$ and $J$ ideals of $A$. Let sa $(I)$ be the set of all $a \in A$ such that $a$ is atomless mod $I$, at $(I)$ the set of all $a \in A$ such that $a$ is atomic $\bmod I$, and $I+J$ the set of all $a \in A$ such that $a=b+c$ for some $b \in I$ and some $c \in J$. Then sa $(I)$, at $(I)$, and $I+J$ are all again ideals of $A$. Let $0^{A}$ be the bottom element of $A$, set $E_{0}=\left\{0^{A}\right\}$, and for every integer $n \geq 0$ set $E_{n+1}=\mathrm{sa}\left(E_{n}\right)+\operatorname{at}\left(E_{n}\right)$.

We now define the Tarski invariants $\operatorname{inv}(A)$ of $A$. If $A$ is the one-element Boolean algebra then $\operatorname{inv}(A)=(-1,0,0)$; if $A \neq E_{n}$ for every $n \geq 0$ then $\operatorname{inv}(A)=(\omega, 0,0)$; otherwise $\operatorname{inv}(A)=\left(i_{1}, i_{2}, i_{3}\right)$ where (1) $A \neq E_{i_{1}}$ and $A=E_{i_{1}+1}$, (2) $i_{2}=0$ if $A / E_{i_{1}}$ is atomic, otherwise $i_{2}=1$, and (3) $i_{3}$ is the number of atoms of $A / E_{i_{1}}$ if that number is finite, and $\omega$ if that number is infinite. Let Inv be the set of triples consisting of $(-1,0,0),(\omega, 0,0)$, and all the triples $\left(i_{1}, i_{2}, i_{3}\right)$ with $i_{1}$ a nonnegative integer, $i_{2} \in\{0,1\}, i_{3}$ a nonnegative integer or $\omega$, and $i_{2}+i_{3}>0$.

For a proof of the following result see [1] or Chapter 7 of [4].

Theorem 2.1. Any two Boolean algebras $A$ and $B$ are elementarily equivalent iff $\operatorname{inv}(A)=\operatorname{inv}(B)$. Moreover, for any $\left(i_{1}, i_{2}, i_{3}\right) \in \operatorname{Inv}$ there is a Boolean algebra $A$ with $\operatorname{inv}(A)=\left(i_{1}, i_{2}, i_{3}\right)$.

3. $P$-spaces. $P$-spaces are a generalization of discrete spaces and were named and studied by Gillman and Henriksen in [2]. Here are two useful examples of nondiscrete $P$-spaces.

ExAmPLE. Let $S$ be an uncountable space in which all points are isolated except for a distinguished point $s$, a neighborhood of $s$ being any set containing $s$ whose complement is countable. Then $S$ is a nondiscrete normal $P$-space and $\operatorname{Clop}(S)$ is an atomic Boolean algebra.

EXAMPLE. A totally ordered set $T$ is called an $\eta_{1}$-set if for any countable subsets $A$ and $B$, with $A<B$, there is a $t \in T$ satisfying $A<t<B$. With the interval topology every $\eta_{1}$-set $T$ is a normal $\mathrm{P}$-space without isolated points and $\operatorname{Clop}(T)$ is an atomless Boolean algebra (see page 193 of [3]).

If $\left\{X_{k}\right\}_{k \in K}$ is an indexed collection of spaces, then we write $\bigoplus_{k \in K} X_{k}$ for their topological sum, i.e. the disjoint union of the $X_{k}$ topologized so that a subset $U$ is open in the sum if and only if its intersection with each $X_{k}$ is open in $X_{k}$. Here are some basic properties of $P$-spaces.

Proposition 3.1. The following hold in any $P$-space $X$ :

(i) Every zero-set of $X$ is open.

(ii) Every subspace of $X$ is a $P$-space. 
(iii) Every completely regular quotient space of $X$ is a $P$-space.

(iv) $X$ is zero-dimensional, i.e., has a base of clopen subsets.

Proof. See pages $62-63$ of [3].

The rest of this paper is devoted to proving Theorem 1.1. Our strategy will be to build for each triple of Tarski invariants a normal $P$-space whose clopen algebra has those invariants. Many of these spaces will be obtained by gluing together in a certain way copies of the two normal $P$-spaces mentioned at the beginning of this section.

4. Topological equivalents of algebraic notions. Let $X$ be an arbitrary normal $P$-space. To simplify our analysis we characterize membership in the Tarski ideals $E_{n}$, at $\left(E_{n}\right)$, and $\operatorname{sa}\left(E_{n}\right)$ of $\operatorname{Clop}(X)$ using a device reminiscent of Cantor-Bendixson derivatives. We associate with $X$ the following descending sequence of closed subspaces: let $X_{0}=X$ and for every integer $n \geq 0$ let

$$
X_{n+1}=\overline{\mathrm{Is}\left(X_{n}\right)} \cap \overline{\left(X_{n}-\overline{\mathrm{Is}\left(X_{n}\right)}\right)}
$$

where the overline represents the topological closure operation in $X$ and Is $\left(X_{n}\right)$ is the set of isolated points of the subspace $X_{n}$.

Proposition 4.1. Each of the following hold for any $G \in \operatorname{Clop}(X)$ and any $n \geq 0$ :

(i) $G \in E_{n}$ if and only if $G \cap X_{n}=\emptyset$.

(ii) $G$ is an atom $\bmod E_{n}$ if and only if $\left|G \cap X_{n}\right|=1$.

(iii) $G \in \operatorname{at}\left(E_{n}\right)$ if and only if $G \cap X_{n} \subseteq \overline{\mathrm{Is}\left(X_{n}\right)}$.

(iv) $G \in \operatorname{sa}\left(E_{n}\right)$ if and only if $G \cap \operatorname{Is}\left(X_{n}\right)=\emptyset$.

Proof. The proof goes by induction on $n$. That (i) holds when $n=0$ is clear. Suppose $G$ is an atom $\bmod E_{0}$. Then $G$ is an atom of $\operatorname{Clop}(X)$, so $G$ must have at least one element. If $G$ has more than one element then because $X$ is Hausdorff and zero-dimensional $G$ has nonempty proper clopen subsets and hence is not an atom of $\operatorname{Clop}(X)$. Thus $\left|G \cap X_{0}\right|=|G|=1$. Conversely, if $\left|G \cap X_{0}\right|=1$ then clearly $G$ is an atom of $\operatorname{Clop}(X)$. Thus (ii) holds when $n=0$. Now $G \in \operatorname{at}\left(E_{0}\right)$ if and only if every clopen subset of $G$ contains an atom of $\operatorname{Clop}(X)$ and hence an isolated point of $X$. Since $X$ is zero-dimensional this is equivalent to having $G \cap X_{0} \subseteq \overline{\operatorname{Is}\left(X_{0}\right)}$. Similarly, $G \in \operatorname{sa}\left(E_{0}\right)$ if and only if $G$ contains no atoms of $\operatorname{Clop}(X)$, which is the same as having $G \cap \operatorname{Is}(X)=\emptyset$. Thus (iii) and (iv) both hold when $n=0$.

Assume the result of the proposition for $n=k \geq 0$ and let $G \in \operatorname{Clop}(X)$. We show that (i)-(iv) hold when $n=k+1$.

Suppose $G \cap X_{k+1}=\emptyset$. Then, by the definition of $X_{k+1}$, we have

$$
\left[G \cap \overline{\mathrm{Is}\left(X_{k}\right)}\right] \cap\left[G \cap \overline{\left(X_{k}-\overline{\operatorname{Is}\left(X_{k}\right)}\right)}\right]=\emptyset .
$$


$X$ is normal so by Urysohn's Lemma (see page 44 of [3]) there is an $f \in C(X)$ such that

$$
G \cap \overline{\mathrm{Is}\left(X_{k}\right)} \subseteq f^{-1}(0)
$$

and

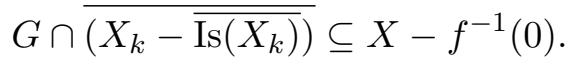

Note that $f^{-1}(0) \in \operatorname{Clop}(X)$ because $X$ is a $P$-space, so $G \cap f^{-1}(0)$ and $G \cap\left(X-f^{-1}(0)\right)$ are also in $\operatorname{Clop}(X)$. If $x \in G \cap X_{k}$ and $x \notin \overline{\operatorname{Is}\left(X_{k}\right)}$ then $x \in X-f^{-1}(0)$ by (2). It follows that $\left(G \cap f^{-1}(0)\right) \cap X_{k} \subseteq \overline{\operatorname{Is}\left(X_{k}\right)}$ and therefore $G \cap f^{-1}(0) \in \operatorname{at}\left(E_{k}\right)$ by the induction hypothesis. If $x \in$ $G \cap \operatorname{Is}\left(X_{k}\right)$ then $x \in f^{-1}(0)$ by $(1)$, so $\left(G \cap\left(X-f^{-1}(0)\right) \cap \operatorname{Is}\left(X_{k}\right)=\emptyset\right.$ and thus $G \cap\left(X-f^{-1}(0)\right) \in \mathrm{sa}\left(E_{k}\right)$ by the induction hypothesis. Hence $G \in \operatorname{at}\left(E_{k}\right)+\operatorname{sa}\left(E_{k}\right)=E_{k+1}$.

Conversely, suppose $G \in E_{k+1}$. Then $G=H \cup F$ for some $H \in \operatorname{sa}\left(E_{k}\right)$ and $F \in$ at $\left(E_{k}\right)$. So by the induction hypothesis $H \cap \operatorname{Is}\left(X_{k}\right)=\emptyset$ and therefore, since $H$ is open in $X, H \cap \overline{\operatorname{Is}\left(X_{k}\right)}=\emptyset$. It follows that $H \cap X_{k+1}=\emptyset$. From the induction hypothesis we also see that $F \cap X_{k} \subseteq \overline{\operatorname{Is}\left(X_{k}\right)}$. So $\left(F \cap X_{k}\right) \cap\left(X_{k}-\overline{\mathrm{Is}\left(X_{k}\right)}\right)=\emptyset$. Since $F$ is open in $X$ it follows that

$$
\left(F \cap X_{k}\right) \cap \overline{\left(X_{k}-\overline{\mathrm{Is}\left(X_{k}\right)}\right)}=\emptyset
$$

and hence that $F \cap X_{k+1}=\emptyset$ as $X_{k+1} \subseteq X_{k}$. Thus $G \cap X_{k+1}=(H \cup F) \cap$ $X_{k+1}=\emptyset$ and we have shown that (i) holds when $n=k+1$.

Suppose $\left|G \cap X_{k+1}\right|=1$. Then $G \notin E_{k+1}$ by (i). Suppose $H \in \operatorname{Clop}(X)$, $H \subseteq G$, and $H \notin E_{k+1}$. Then $H \cap X_{k+1} \neq \emptyset$ by (i). Since $\left|G \cap X_{k+1}\right|=1$ and $H \subseteq G, G$ and $H$ must contain the same member of $X_{k+1}$. Therefore $(G-H) \cap X_{k+1}=\emptyset$ and so $G-H \in E_{k+1}$ by (i). Hence $G$ is an atom $\bmod E_{k+1}$. Conversely, suppose $G$ is an atom $\bmod E_{k+1}$. Then $G \notin E_{k+1}$ so $\left|G \cap X_{k+1}\right| \geq 1$ by (i). If there were more than one member of $X_{k+1}$ in $G$ then it would follow from (i) and the fact that $X$ is Hausdorff and zero-dimensional that $G$ is not an atom $\bmod E_{k+1}$, which contradicts our supposition. Hence $\left|G \cap X_{k+1}\right|=1$ and (ii) holds when $n=k+1$.

Suppose $G \cap X_{k+1} \subseteq \overline{\overline{\mathrm{Is}}\left(X_{k+1}\right)}$. Suppose $H \in \operatorname{Clop}(X), H \subseteq G$, and $H \notin E_{k+1}$. Then $H \cap X_{k+1} \neq \emptyset$ by (i). Since $H \subseteq G$ and $H$ is open in $X$ it follows that $H \cap \operatorname{Is}\left(X_{k+1}\right) \neq \emptyset$. So there is an $F \in \operatorname{Clop}(X)$ such that $\left|(F \cap H) \cap X_{k+1}\right|=1$. By (ii), $F \cap H$ is an atom $\bmod E_{k+1}$. Thus $G \in \operatorname{at}\left(E_{k+1}\right)$. Conversely, suppose $G \in \operatorname{at}\left(E_{k+1}\right)$ and $x \in G \cap X_{k+1}$. Let $H$ be an open neighborhood of $x$ in $X$. Since $X$ is zero-dimensional we may assume that $H \in \operatorname{Clop}(X)$. By (i), $H \cap G \notin E_{k+1}$ and, since $G \in \operatorname{at}\left(E_{k+1}\right)$, there must be an $F \in \operatorname{Clop}(X)$ such that $F \subseteq H \cap G$ and $F$ is an atom $\bmod$ $E_{k+1}$. By (ii), $F \cap X_{k+1}$ has only one element which is therefore an isolated 
point of $X_{k+1}$. Since this point is in $H$, it follows that $G \cap X_{k+1} \subseteq \overline{\operatorname{Is}\left(X_{k+1}\right)}$. Hence (iii) holds when $n=k+1$.

Finally, $G \cap \operatorname{Is}\left(X_{k+1}\right) \neq \emptyset$ if and only if there is an $H \in \operatorname{Clop}(X)$ such that $H \subseteq G$ and $\left|H \cap X_{k+1}\right|=1$. By (i) and (ii) this is equivalent to $H$ being an atom $\bmod E_{k+1}$, which means that $G \notin \mathrm{sa}\left(E_{k+1}\right)$. Hence (iv) holds for $n=k+1$.

REMARK. Inspection of the proof of Proposition 4.1 reveals that the result holds for any zero-dimensional Hausdorff space in which disjoint closed sets may be separated by disjoint clopen sets. The latter property is also possessed by zero-dimensional Lindelöf spaces (see page 247 of [3]). So, for example, Proposition 4.1 holds in any Boolean space.

Proposition 4.2. Each of the following hold for any integers $n, m \geq 0$ :

(i) $\operatorname{inv}(\operatorname{Clop}(X))=(n, 0, m)$ iff $\left|X_{n}\right|=m>0$.

(ii) $\operatorname{inv}(\operatorname{Clop}(X))=(n, 0, \omega)$ iff $\operatorname{Is}\left(X_{n}\right)$ is infinite and $X_{n} \subseteq \overline{\operatorname{Is}\left(X_{n}\right)}$.

(iii) $\operatorname{inv}(\operatorname{Clop}(X))=(n, 1, m)$ iff $\left|\operatorname{Is}\left(X_{n}\right)\right|=m$ and $X_{n} \nsubseteq \overline{\operatorname{Is}\left(X_{n}\right)}$.

(iv) $\operatorname{inv}(\operatorname{Clop}(X))=(n, 1, \omega)$ iff $\operatorname{Is}\left(X_{n}\right)$ is infinite and $X_{n} \nsubseteq \overline{\operatorname{Is}\left(X_{n}\right)}$.

(v) $\operatorname{inv}(\operatorname{Clop}(X))=(\omega, 0,0)$ iff $X_{k} \neq \emptyset$ for all $k \geq 0$.

Proof. By Proposition 4.1, $X_{k} \neq \emptyset$ for all $k \geq 0$ if and only if $E_{k} \neq$ $\operatorname{Clop}(X)$ for all $k \geq 0$. Thus (v) holds.

Fix $n, m \geq 0$. Suppose $\operatorname{inv}(\operatorname{Clop}(X))=(n, 0, m)$. Then $E_{n} \neq \operatorname{Clop}(X)$ and $\operatorname{Clop}(X) / E_{n}$ is atomic with $m$ atoms. Note that $m>0$, for otherwise $\operatorname{Clop}(X)=E_{n}$. Since $\operatorname{Clop}(X) / E_{n}$ is atomic, $X \in \operatorname{at}\left(E_{n}\right)$ and so $X_{n}=$ $\overline{\mathrm{Is}}\left(X_{n}\right)$ follows from Proposition 4.1. Say $G_{1}, \ldots, G_{m}$ are representatives of the $m$ atoms $\bmod E_{n}$. By Proposition $4.1,\left|G_{i} \cap X_{n}\right|=1$ for all $i$. So each $G_{i}$ contains an isolated point of $X_{n}$. If $G_{i} \cap G_{j} \cap X_{n} \neq \emptyset$ for some $i \neq j$, then $G_{i} \cap G_{j} \notin E_{n}$ by Proposition 4.1, which contradicts the fact that $G_{i}$ and $G_{j}$ represent distinct atoms $\bmod E_{n}$. Thus $\left|\operatorname{Is}\left(X_{n}\right)\right| \geq m$. Now $\operatorname{Clop}(X) / E_{n}$ is atomic, so $\bigcup_{i=1}^{m} G_{i}$ is equal to $X$ modulo $E_{n}$ and therefore $X-\bigcup_{i=1}^{m} G_{i}$ contains no points of $X_{n}$ by Proposition 4.1. Hence $m=\left|\operatorname{Is}\left(X_{n}\right)\right|=\left|X_{n}\right|$.

Conversely, suppose $\left|X_{n}\right|=m>0$. Then $\left|\operatorname{Is}\left(X_{n}\right)\right|=m>0$ and $X_{n} \subseteq \overline{\operatorname{Is}\left(X_{n}\right)}$. So $X \in$ at $\left(E_{n}\right)$ by Proposition 4.1 and therefore $\operatorname{Clop}(X) / E_{n}$ is atomic and the first Tarski invariant of $\operatorname{Clop}(X)$ is at most $n$. Since $\left|\operatorname{Is}\left(X_{n}\right)\right|=m>0, X_{n} \neq \emptyset$ and $X \notin E_{n}$ by Proposition 4.1. Hence $E_{n} \neq \operatorname{Clop}(X)$ and the first two Tarski invariants of $\operatorname{Clop}(X)$ are $n$ and 0. Now using the fact that $X$ is Hausdorff and zero-dimensional we may find pairwise disjoint $G_{1}, \ldots, G_{m} \in \operatorname{Clop}(X)$ such that $\left|G_{i} \cap X_{n}\right|=1$ for each $i$. It follows from Proposition 4.1 that there are at least $m$ atoms modulo $E_{n}$. Suppose $G \in \operatorname{Clop}(X)$ is an atom $\bmod E_{n}$. By Proposition 4.1, $G$ must contain exactly one member of $X_{n}$ and therefore must be equal to one of the 
$G_{i}$ modulo $E_{n}$. Thus $\operatorname{Clop}(X) / E_{n}$ has exactly $m$ atoms, the third invariant of $\operatorname{Clop}(X)$ is $m$, and (i) holds.

From the proof of (i) we see that having at least $m$ atoms mod $E_{n}$ is equivalent to $\left|\operatorname{Is}\left(X_{n}\right)\right| \geq m$. With the aid of this fact, (ii)-(iv) are easily established.

5. Technical topological lemmas. Let $\left\{Y_{k}\right\}_{k \in K}$ be a collection of disjoint spaces, $X$ a space disjoint from all the $Y_{k}$ and of cardinality at least $|K|, y_{k} \in Y_{k}$ for each $k \in K$, and $\left\{x_{k}\right\}_{k \in K}$ a collection of distinct points in $X$. We call $Z$ the pointwise gluing of $\left\{\left(Y_{k}, y_{k}\right)\right\}_{k \in K}$ and $\left(X,\left\{x_{k}\right\}_{k \in K}\right)$ over $K$ if

$$
Z=\left(\left(\bigoplus_{k \in K} Y_{k}\right) \oplus X\right) / \sim
$$

where $\sim$ is the equivalence relation on $\left(\bigoplus_{k \in K} Y_{k}\right) \oplus X$ which identifies $y_{k}$ with $x_{k}$ for each $k \in K$. We call the $y_{k}$ and $x_{k}$ glue points and, for any $U \subseteq\left(\bigoplus_{k \in K} Y_{k}\right) \oplus X$, we write $\operatorname{gp}(U)$ for $\left\{k \in K:\left\{x_{k}, y_{k}\right\} \cap U \neq \emptyset\right\}$. Finally, we always use $q$ to denote the canonical quotient map from $\left(\bigoplus_{k \in K} Y_{k}\right) \oplus X$ to $Z$.

Lemma 5.1. Let $Z$ be the pointwise gluing of the spaces $\left\{\left(Y_{k}, y_{k}\right)\right\}_{k \in K}$ and $\left(X,\left\{x_{k}\right\}_{k \in K}\right)$ over $K$. If $U \subseteq W=\left(\bigoplus_{k \in K} Y_{k}\right) \oplus X$ and $\operatorname{gp}(U) \cap$ $\operatorname{gp}(W-U)=\emptyset$ then (i) $U$ is open in $W$ if and only if $q(U)$ open in $Z$, and (ii) $U$ is closed in $W$ if and only if $q(U)$ is closed in $Z$.

Proof. Since $\operatorname{gp}(U) \cap \operatorname{gp}(W-U)=\emptyset$, we see that $q^{-1}(q(U))=U$. Therefore $U$ is open if and only if $q^{-1}(q(U))$ is open. But the latter is open just in case $q(U)$ is open, because $q$ is a quotient map. Thus (i) holds.

By (i), $U$ is closed if and only if $q(W-U)$ is open. $\operatorname{As} g p(U) \cap \operatorname{gp}(W-U)$ $=\emptyset$ we see that $q(W-U)=q(W)-q(U)$. But $q(W)-q(U)$ is open if and only if $q(U)$ is closed because $q$ is surjective. Hence (ii) holds.

Lemma 5.2. Let $Z$ be the pointwise gluing of the normal $P$-spaces $\left\{\left(Y_{k}, y_{k}\right)\right\}_{k \in K}$ and $\left(X,\left\{x_{k}\right\}_{k \in K}\right)$ over $K$. Then $Z$ is a normal P-space.

Proof. Since any topological sum of $P$-spaces is a $P$-space and since any completely regular quotient of a $P$-space is a $P$-space (see page 63 of [3]), to prove the lemma it suffices to show that $Z$ is normal. Let $F$ and $G$ be disjoint closed subsets of $Z$. Then $q^{-1}(F)$ and $q^{-1}(G)$ are disjoint closed sets in $W=\left(\bigoplus_{k \in K} Y_{k}\right) \oplus X$. So

$$
q^{-1}(F)=\left(\bigcup_{k \in K} F_{k}\right) \cup H_{F} \quad \text { and } \quad q^{-1}(G)=\left(\bigcup_{k \in K} G_{k}\right) \cup H_{G},
$$


where $F_{k}, G_{k}$ are disjoint closed sets in $Y_{k}$ for each $k$, and $H_{F}$ and $H_{G}$ are disjoint closed sets in $X$. Note that

$$
y_{k} \in F_{k} \quad \text { iff } \quad x_{k} \in H_{F}
$$

and

$$
y_{k} \in G_{k} \quad \text { iff } \quad x_{k} \in H_{G}
$$

for each $k \in K$. Since $X$ and the $Y_{k}$ 's are normal, there exist disjoint open $U, V \subseteq X$ and disjoint open $U_{k}, V_{k} \subseteq Y_{k}$ such that

$$
q^{-1}(F) \subseteq\left(\bigcup_{k \in K} U_{k}\right) \cup U \text { and } q^{-1}(G) \subseteq\left(\bigcup_{k \in K} V_{k}\right) \cup V .
$$

In order to ensure that their images under $q$ will be open, we may need to adjust these open sets separating $q^{-1}(F)$ and $q^{-1}(G)$. First, since $F_{k}$ and $G_{k}$ are closed and $Y_{k}$ is completely regular, we may assume that

$$
y_{k} \in \bar{U}_{k} \quad \text { iff } \quad y_{k} \in F_{k}
$$

and

$$
y_{k} \in \bar{V}_{k} \quad \text { iff } \quad y_{k} \in G_{k}
$$

for each $k \in K$. Next, let $L=\left\{k \in K: x_{k} \in U-H_{F}\right\}$ and $M=\{k \in K$ : $\left.x_{k} \in V-H_{G}\right\}$. Since $U \cap V=\emptyset$ and $H_{G} \subseteq U$, if $k \in L$ then $x_{k} \notin H_{F} \cup H_{G}$ and so $y_{k} \notin \bar{U}_{k} \cup \bar{V}_{k}$ by (1)-(4). Since each $Y_{k}$ is completely regular we therefore may choose for each $k \in L$ an open $T_{k} \subseteq Y_{k}$ such that $y_{k} \in T_{k}$ and $T_{k} \cap\left(U_{k} \cup V_{k}\right)=\emptyset$. Similarly, we may choose for each $k \in M$ an open $S_{k} \subseteq Y_{k}$ with $y_{k} \in S_{k}$ and $S_{k} \cap\left(U_{k} \cup V_{k}\right)=\emptyset$. Finally, set

$$
\begin{aligned}
& O_{F}=\left(\bigcup_{k \in K} U_{k}\right) \cup U \cup\left(\bigcup_{k \in L} T_{k}\right), \\
& O_{G}=\left(\bigcup_{k \in K} V_{k}\right) \cup V \cup\left(\bigcup_{k \in M} S_{k}\right) .
\end{aligned}
$$

We will show that $q\left(O_{F}\right)$ and $q\left(O_{G}\right)$ separate $F$ and $G$ in $Z$.

First, clearly $F \subseteq q\left(O_{F}\right)$ and $G \subseteq q\left(O_{G}\right)$. Next we show that $q\left(O_{F}\right)$ and $q\left(O_{G}\right)$ are open in $Z$. Fix $k \in K$. Then from the definition of $O_{F}$ we see that $x_{k} \in O_{F}$ if and only if $x_{k} \in U$. Now $x_{k} \in U$ means $x_{k} \in H_{F}$ or $x_{k} \in U-H_{F}$, so by (1) and the definition of $T_{k}$ we have $x_{k} \in U$ if and only if either $y_{k} \in F_{k}$ or $y_{k} \in T_{k}$. Thus $x_{k} \in O_{F}$ is equivalent to $y_{k} \in O_{F}$. It follows that $\operatorname{gp}\left(O_{F}\right) \cap \operatorname{gp}\left(W-O_{F}\right)=\emptyset$ and so $q\left(O_{F}\right)$ is open in $Z$ by Lemma 5.1. A similar argument shows that $\operatorname{gp}\left(O_{G}\right) \cap \operatorname{gp}\left(W-O_{G}\right)=\emptyset$ and so $q\left(O_{G}\right)$ is also open in $Z$. Finally, we show that $q\left(O_{F}\right) \cap q\left(O_{G}\right)=\emptyset$. Since $\operatorname{gp}\left(O_{F}\right) \cap \operatorname{gp}\left(W-O_{F}\right)=\operatorname{gp}\left(O_{G}\right) \cap \operatorname{gp}\left(W-O_{G}\right)=\emptyset$ we see that $q\left(O_{F}\right) \cap q\left(O_{G}\right) \neq \emptyset$ implies $O_{F} \cap O_{G} \neq \emptyset$. So to complete the proof of the 
lemma it suffices to show that $O_{F} \cap O_{G}=\emptyset$. Let $w \in O_{F}$. We show that $w \notin O_{G}$.

CASE (1). Suppose $w \in U_{t}$ for some $t \in K$. Then $w \in Y_{t}$ and $Y_{t} \cap X=\emptyset$, so $w \notin V$. Since $Y_{k} \cap Y_{t}=\emptyset$ whenever $k \neq t$, and since $U_{t} \cap V_{t}=\emptyset$, we see that $w \notin \bigcup_{k \in K} V_{k}$. Fix $k \in M$. If $t \neq k$ then $w \notin S_{k}$ because $w \in Y_{t}, S_{k} \subseteq Y_{k}$, and $Y_{t} \cap Y_{k}=\emptyset$. If $t=k$ then $w \notin V_{k}$ because $w \in U_{t}$ and $S_{k} \cap U_{k}=\emptyset$ by choice of $S_{k}$. Thus $w \notin \bigcup_{k \in M} S_{k}$. Hence $w \notin O_{G}$.

CASE (2). Suppose $w \in U$. Then $w \in X$ so $w \notin\left(\bigcup_{k \in K} V_{k}\right) \cup\left(\bigcup_{k \in M} S_{k}\right)$ because $X \cap\left(\bigcup_{k \in K} Y_{k}\right)=\emptyset$. Also, $U \cap V=\emptyset$ so $w \notin V$. Hence $w \notin O_{G}$.

CASE (3). Suppose $w \in T_{l}$ for some $l \in L$. Then $w \notin V$ since $T_{l} \subseteq Y_{l}$ and $Y_{l} \cap X=\emptyset$. Now $T_{l} \cap Y_{k}=\emptyset$ whenever $l \neq k$, and $T_{l} \cap V_{l}=\emptyset$ by choice of $T_{l}$, so we see that $w \notin \bigcup_{k \in K} V_{k}$. Finally, $w \notin \bigcup_{k \in M} S_{k}$ because $L \cap M=\emptyset$ and so $T_{l} \cap S_{k}=\emptyset$ for any $k \in M$. Hence $w \notin O_{G}$.

Lemma 5.3. Let $X$ be a normal P-space and $U \in \operatorname{Clop}(X)$. Then each of the following holds for any $n \geq 0$ :

(i) $U_{n}=U \cap X_{n}$.

(ii) $\operatorname{Is}\left(U_{n}\right)=U \cap \operatorname{Is}\left(X_{n}\right)$.

(iii) $U_{n}-\overline{\mathrm{Is}\left(U_{n}\right)}=U \cap\left(X_{n}-\overline{\mathrm{Is}\left(X_{n}\right)}\right)$.

Proof. The proof goes by induction on $n$. Note that with the subspace topology $U$ is a normal $P$-space because it is a closed subset of $X$. Suppose $n=0$. Then $X_{n}=X$ and $U_{n}=U$, so (i) is obvious and (ii) holds because $U \in \operatorname{Clop}(X)$. If $G$ is an open set in $U$ with no isolated points of $U$, then $G$ is open in $X$ and contains no isolated points of $X$ by (ii). Similarly, if $G$ is an open set in $X$ with no isolated points then $G \cap U$ is an open set in $U$ with no isolated points in $U$ by (ii). Thus (iii) holds when $n=0$.

Now suppose the result holds for $n=k \geq 0$. By definition

$$
U_{k+1}=\overline{\mathrm{Is}\left(U_{k}\right)} \cap \overline{\left(U_{k}-\overline{\mathrm{Is}\left(U_{k}\right)}\right)}
$$

and by the induction hypothesis

$$
\overline{\operatorname{Is}\left(U_{k}\right)} \cap \overline{\left(U_{k}-\overline{\operatorname{Is}\left(U_{k}\right)}\right)}=\overline{\left(U \cap \operatorname{Is}\left(X_{k}\right)\right)} \cap \overline{\left(U \cap\left(X_{k}-\overline{\operatorname{Is}\left(X_{k}\right)}\right)\right.} .
$$

Now $U$ is clopen in $X$, so

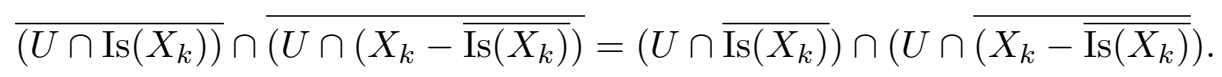

Since the right-hand side of the last formula is equal to $U \cap X_{k+1}$, we see that (i) holds for $n=k+1$. That (ii) holds when $n=k+1$ follows easily from (i) and the fact that $U \in \operatorname{Clop}(X)$.

Finally,

$$
U_{k+1}-\overline{\operatorname{Is}\left(U_{k+1}\right)}=\left(U \cap X_{k+1}\right)-\left(\overline{U \cap \operatorname{Is}\left(X_{k+1}\right)}\right)
$$


by (i) and (ii), and since $U$ is clopen in $X$,

$$
\left(U \cap X_{k+1}\right)-\left(\overline{U \cap \operatorname{Is}\left(X_{k+1}\right)}\right)=\left(U \cap X_{k+1}\right)-\left(U \cap \overline{\operatorname{Is}\left(X_{k+1}\right)}\right) .
$$

Since the right-hand side of the last formula is equal to $U \cap\left(X_{k+1}-\overline{\mathrm{Is}\left(X_{k+1}\right)}\right)$, (iii) holds when $n=k+1$.

Lemma 5.4. Let $W$ and $Z$ be normal $P$-spaces and $q: W \rightarrow Z$ a map. If $U \in \operatorname{Clop}(W), q(U) \in \operatorname{Clop}(Z)$, and $q \mid U$ is a homeomorphism, then each of the following hold for any $n \geq 0$ :

(i) $q\left(U \cap W_{n}\right)=q(U) \cap Z_{n}$.

(ii) $q\left(U \cap \operatorname{Is}\left(W_{n}\right)\right)=q(U) \cap \operatorname{Is}\left(Z_{n}\right)$.

(iii) $q\left(U \cap\left(W_{n}-\overline{\mathrm{Is}\left(W_{n}\right)}\right)\right)=q(U) \cap\left(Z_{n}-\overline{\mathrm{Is}\left(Z_{n}\right)}\right)$.

Proof. The result essentially follows from Lemma 5.3. For example, $q\left(U \cap W_{n}\right)=q\left(U_{n}\right)$ by Lemma 5.3 since $U \in \operatorname{Clop}(W)$. Then $q\left(U_{n}\right)=q(U)_{n}$ because $q \mid U$ is a homeomorphism. Finally, $q(U) \in \operatorname{Clop}(Z)$ so $q(U)_{n}=$ $q(U) \cap Z_{n}$ by Lemma 5.3. Similar arguments prove the other two identities.

Lemma 5.5. Suppose $\left\{Y_{k}\right\}_{k \in K}$ is a collection of normal P-spaces. If $W=\bigoplus_{k \in K} Y_{k}$, then each of the following hold for any $n \geq 0$ :

(i) $W_{n}=\bigcup_{k \in K}\left(Y_{k}\right)_{n}$.

(ii) $\operatorname{Is}\left(W_{n}\right)=\bigcup_{k \in K} \operatorname{Is}\left(\left(Y_{k}\right)_{n}\right)$.

(iii) $W_{n}-\overline{\mathrm{Is}\left(W_{n}\right)}=\bigcup_{k \in K}\left(\left(Y_{k}\right)_{n}-\overline{\mathrm{Is}\left(\left(Y_{k}\right)_{n}\right)}\right)$.

Proof. Since $Y_{k} \in \operatorname{Clop}(W)$ for all $k \in K$, we can apply Lemma 5.3.

Lemma 5.6. Let $Z$ be the pointwise gluing of the normal $P$-spaces $\left\{\left(Y_{k}, y_{k}\right)\right\}_{k \in K}$ and $\left(X,\left\{x_{k}\right\}_{k \in K}\right)$ over $K$. Then $q\left(\left(Y_{k}\right)_{n}\right) \subseteq Z_{n}$ for every $k \in K$ and every $n \geq 0$.

Proof. Let $W=\left(\bigoplus_{k \in K} Y_{k}\right) \oplus X$. Note that $Z$ is a normal $P$-space by Lemma 5.2. Fix $k \in K$. If $n=0$ then the result holds because $\left(Y_{k}\right)_{0}=Y_{k}$ and $Z_{0}=Z$. Suppose $n>0$ and $w \in\left(Y_{k}\right)_{n}$. Let $V$ be a neighborhood of $q(w)$ in $Z$. We must show that $V$ meets both $\operatorname{Is}\left(Z_{n-1}\right)$ and $Z_{n-1}-\overline{\operatorname{Is}\left(Z_{n-1}\right)}$. Now $w \in\left(Y_{k}\right)_{n}$ and $n>0$, so we know that

$$
w \in \overline{\mathrm{Is}\left(\left(Y_{k}\right)_{n-1}\right)} \cap \overline{\left(\left(Y_{k}\right)_{n-1}-\overline{\mathrm{Is}\left(\left(Y_{k}\right)_{n-1}\right)}\right)} .
$$

Therefore $q^{-1}(V) \cap Y_{k}$ must contain infinitely many elements of $\operatorname{Is}\left(\left(Y_{k}\right)_{n-1}\right)$ and infinitely many elements of $\left(Y_{k}\right)_{n-1}-\overline{\mathrm{Is}\left(\left(Y_{k}\right)_{n-1}\right)}$. Pick $y \in \operatorname{Is}\left(\left(Y_{k}\right)_{n-1}\right) \cap$ $\left(q^{-1}(V) \cap Y_{k}\right)$ such that $y \neq y_{k}$.

Choose $U \in \operatorname{Clop}(W)$ such that $U \subseteq Y_{k}, y \in U$, and $y_{k} \notin U$. Then $\operatorname{gp}(U)=\emptyset$, so $U$ satisfies the hypotheses of Lemma 5.4. Since $y \in \operatorname{Is}\left(W_{n-1}\right)$ by Lemma 5.5 , it follows that $q(y) \in \operatorname{Is}\left(Z_{n-1}\right)$. A similar argument shows that $V$ contains members of $Z_{n-1}-\overline{\mathrm{Is}\left(Z_{n-1}\right)}$. 


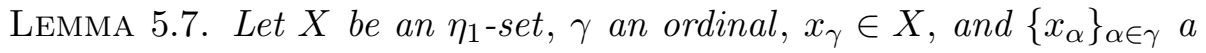
strictly increasing sequence in $X$ that is cofinal in $\left\{x \in X: x<x_{\gamma}\right\}$. If $Z$ is the pointwise gluing of the normal $P$-spaces $\left\{\left(Y_{\alpha}, y_{\alpha}\right)\right\}_{\alpha \in \gamma}$ and $\left(X,\left\{x_{\alpha}\right\}_{\alpha \in \gamma}\right)$ over $\gamma, n \geq 1$, and $\left(Y_{\alpha}\right)_{n}=\left\{y_{\alpha}\right\}$ for all $\alpha \in \gamma$, then $q\left(x_{\gamma}\right) \in \overline{\operatorname{Is}\left(Z_{n}\right)}-\operatorname{Is}\left(Z_{n}\right)$ and $Z_{n+1}=\emptyset$.

Proof. Let $W=\left(\bigoplus_{\alpha \in \gamma} Y_{\alpha}\right) \oplus X$. Note that $Z$ is a normal $P$-space by Lemma 5.2 and fix $\alpha<\gamma$. First we show that $q\left(y_{\alpha}\right) \in \overline{\mathrm{Is}\left(Z_{n}\right)}$.

CASE (1). Suppose there is an open set $G \subseteq X$ such that $x_{\alpha} \in G$ and $\operatorname{gp}(G)=\{\alpha\}$. We show that $q\left(y_{\alpha}\right) \in \operatorname{Is}\left(Z_{n}\right)$. By hypothesis $y_{\alpha} \in\left(Y_{\alpha}\right)_{n}$, so $q\left(y_{\alpha}\right) \in Z_{n}$ by Lemma 5.6. Since $W$ is zero-dimensional we may find $H \in$ $\operatorname{Clop}(W)$ such that $x_{\alpha}, y_{\alpha} \in H$ and $H \subseteq G \cup Y_{\alpha}$. Note that $\operatorname{gp}(H)=\{\alpha\}$ and $\operatorname{gp}(H) \cap \operatorname{gp}(W-H)=\emptyset$. So $q(H) \in \operatorname{Clop}(Z)$ by Lemma 5.1. We claim that $q(H) \cap Z_{n}=\left\{q\left(y_{\alpha}\right)\right\}$. Let $w \in H$. We already know that $q\left(y_{\alpha}\right)=q\left(x_{\alpha}\right) \in Z_{n}$, so suppose $w$ is not $y_{\alpha}$ or $x_{\alpha}$. Then there is a $U \in \operatorname{Clop}(W)$ such that $w \in U \subseteq H$ and $x_{\alpha}, y_{\alpha} \notin U$. Now $X$ is an $\eta_{1}$-set and $n \geq 1$, so $X_{n}=\emptyset$, and $\left(Y_{\alpha}\right)_{n}=\left\{y_{\alpha}\right\}$. It follows that $U \cap\left(Y_{\alpha}\right)_{n}=U \cap X_{n}=\emptyset$ and hence that $U \cap W_{n}=\emptyset$, by Lemma 5.5. Since $\operatorname{gp}(U)=\emptyset$, we may apply Lemma 5.4 to conclude that $q(U) \cap Z_{n}=\emptyset$. Thus $q(w) \notin Z_{n}, q(H) \cap Z_{n}=\left\{q\left(y_{\alpha}\right)\right\}$, and $q\left(y_{\alpha}\right) \in \overline{\mathrm{Is}\left(Z_{n}\right)}$.

CASE (2). Suppose the hypothesis of Case (1) fails. Let $V$ be an open neighborhood of $q\left(y_{\alpha}\right)$ in $Z$. Then $q^{-1}(V) \cap X$ is open in $X$, and since $X$ is an $\eta_{1}$-set, there is an open interval $I \subseteq q^{-1}(V) \cap X$ such that $x_{\alpha} \in I$. By our supposition there is a $\beta<\gamma$ such that $\beta \neq \alpha$ and $x_{\beta} \in I$. Since either $x_{\alpha+1}$ or $x_{\beta+1}$ is in $I$, we may choose a successor ordinal $\delta<\gamma$ such that $x_{\delta} \in I$. Then $\left(x_{\delta-1}, x_{\delta+1}\right) \cap I$ is an open subset of $X$ containing $x_{\delta}$ whose only glue point is $x_{\delta}$, so $q\left(x_{\delta}\right) \in \operatorname{Is}\left(Z_{n}\right)$ by Case (1). Since $q\left(x_{\delta}\right) \in V$, it follows that $q\left(y_{\alpha}\right) \in \overline{\mathrm{Is}\left(Z_{n}\right)}$.

Now we show that $q\left(x_{\gamma}\right) \in \overline{\operatorname{Is}\left(Z_{n}\right)}-\operatorname{Is}\left(Z_{n}\right)$. Let $G \in \operatorname{Clop}(Z)$ be a neighborhood of $q\left(x_{\gamma}\right)$. Then $q^{-1}(G) \cap X$ is open in $X$, and since $X$ is an $\eta_{1}$-set, there is an open interval $U \subseteq q^{-1}(G) \cap X$ containing $x_{\gamma}$. Since $U$ is an open interval there must be an $x \in U$ such that $x<x_{\gamma}$. But $\left\{x_{\alpha}\right\}_{\alpha<\gamma}$ is cofinal in $\left\{x \in X: x<x_{\gamma}\right\}$, so $x_{\alpha} \in U$ for some $\alpha<\gamma$. As we know, $q\left(x_{\alpha}\right) \in \overline{\mathrm{Is}\left(Z_{n}\right)}$ and hence $G$ must contain some member of $\operatorname{Is}\left(Z_{n}\right)$. Since $G$ was arbitrary and $q\left(x_{\alpha}\right) \neq q\left(x_{\gamma}\right)$, it follows that $q\left(x_{\gamma}\right) \in \overline{\operatorname{Is}\left(Z_{n}\right)}-\operatorname{Is}\left(Z_{n}\right)$.

Finally, to prove that $Z_{n+1}=\emptyset$ we show that $Z_{n}-\overline{\operatorname{Is}\left(Z_{n}\right)}=\emptyset$. Let $w \in W$. If $w=y_{\alpha}$ for some $\alpha \in \gamma$ then $q(w) \in \overline{\mathrm{Is}\left(Z_{n}\right)}$. If $w \in Y_{\alpha}$ for some $\alpha \in \gamma$ and $w \neq y_{\alpha}$ then $w \notin\left(Y_{\alpha}\right)_{n}$, and so $q(w) \notin Z_{n}$ follows from Lemma 5.4. If $w \in X$ and there is $U \in \operatorname{Clop}(X)$ such that $\operatorname{gp}(U)=\emptyset$ then $q(w) \notin Z_{n}$ follows from Lemma 5.4. Otherwise, every neighborhood of $w$ contains some $x_{\alpha}$ and therefore $q(w) \in \overline{\mathrm{Is}\left(Z_{n}\right)}$. Thus $Z_{n}-\overline{\operatorname{Is}\left(Z_{n}\right)}=\emptyset$. 
Lemma 5.8. Let $Z$ be the pointwise gluing of the normal $P$-spaces $\left(Y, y_{0}\right)$ and $\left(X, x_{0}\right)$ and let $n \geq 0$. If $Y_{n+1}=\emptyset=X_{n+1}, y_{0} \in \overline{\operatorname{Is}\left(Y_{n}\right)}-\operatorname{Is}\left(Y_{n}\right)$, and $x_{0} \in X_{n}-\overline{\operatorname{Is}\left(X_{n}\right)}$, then $\operatorname{inv}(\operatorname{Clop}(Z))=(n+1,0,1)$.

Proof. Let $W=X \oplus Y$. We know that $Z$ is a normal $P$-space by Lemma 5.2. Let $z_{0}=q\left(x_{0}\right)=q\left(y_{0}\right)$. By Proposition 4.2, it suffices to show that $Z_{n+1}=\left\{z_{0}\right\}$. First we show that $z_{0} \in Z_{n+1}$. Let $V$ be any open neighborhood of $z_{0}$ in $Z$. Since $Z$ is zero-dimensional we may assume that $V \in \operatorname{Clop}(Z)$. Since $y_{0} \in q^{-1}(V)$ and $y_{0} \in \overline{\operatorname{Is}\left(Y_{n}\right)}-\operatorname{Is}\left(Y_{n}\right)$, we may pick $y \in\left(q^{-1}(V) \cap Y\right) \cap \operatorname{Is}\left(Y_{n}\right)$ such that $y \neq y_{0}$. Then $y \in \operatorname{Is}\left(W_{n}\right)$ by Lemma 5.5 and there is a clopen subset $U \subseteq Y$ with $y \in U$ and $y_{0} \notin U$. Since $\operatorname{gp}(U)=\emptyset$, it follows from Lemma 5.4 that $q(y) \in \operatorname{Is}\left(Z_{n}\right)$. Thus $V \cap \operatorname{Is}\left(Z_{n}\right) \neq \emptyset$ and $z_{0} \in \overline{\mathrm{Is}\left(Z_{n}\right)}$.

Now $q^{-1}(V) \cap X \in \operatorname{Clop}(X)$ and by hypothesis $x_{0} \notin \overline{\operatorname{Is}\left(X_{n}\right)}$, so there is an $H \in \operatorname{Clop}(X)$ with $x_{0} \in H \subseteq\left(q^{-1}(V) \cap X\right) \cap\left(X_{n}-\overline{\operatorname{Is}\left(X_{n}\right)}\right)$. Since $x_{0} \in H$ and $x_{0} \notin \operatorname{Is}\left(X_{n}\right)$, we may choose $x \in H$ with $x \neq x_{0}$. Then $x \in W_{n}-\overline{\operatorname{Is}\left(W_{n}\right)}$ by Lemma 5.5. Let $U$ be a clopen subset of $X$ containing $x$ but not $x_{0}$. Then $\operatorname{gp}(U)=\emptyset$, so $q(x) \in Z_{n}-\overline{\mathrm{Is}\left(Z_{n}\right)}$ by Lemma 5.4. Thus $V \cap\left(Z_{n}-\overline{\operatorname{Is}\left(Z_{n}\right)}\right) \neq \emptyset$ and $z_{0} \in \overline{Z_{n}-\overline{\mathrm{Is}\left(Z_{n}\right)}}$. Hence $z_{0} \in Z_{n+1}$.

Finally, we show that $z_{0}$ is the only member of $Z_{n+1}$. Suppose $w \in W$ with $x_{0} \neq w \neq y_{0}$. Then there is a clopen neighborhood $U$ of $w$ in $W$ such that $x_{0}, y_{0} \notin U$. Since $Y_{n+1}=X_{n+1}=\emptyset$, Lemma 5.5 tells us that $W_{n+1}=\emptyset$. Thus $w \notin W_{n+1}$. But $\operatorname{gp}(U)=\emptyset$, so it follows from Lemma 5.4 that $q(w) \notin Z_{n+1}$.

6. Construction of $P$-spaces. The empty space is a normal $P$-space whose algebra of clopen subsets has invariants $(-1,0,0)$. Let $X$ be any one-point space. Then $X$ is a normal $P$-space and $\left|X_{0}\right|=|X|=1$. So it follows from Proposition 4.2 that $\operatorname{inv}(\operatorname{Clop}(X))=(0,0,1)$. Next let $Y$ be the space from the first example at the start of Section 2 and let $X$ be an $\eta_{1}$-set. Let $y_{0}$ be the sole nonisolated point in $Y$ and pick some $x_{0}$ in $X$. Note that $\operatorname{Is}(X)=\emptyset$ and that $\operatorname{Is}(Y)=Y-\left\{y_{0}\right\}$. Form $Z$, the pointwise gluing of $\left(Y, y_{0}\right)$ and $\left(X, x_{0}\right)$. Then $Z$ is a normal $P$-space by Lemma 5.2. Since $Y_{1}=\emptyset=X_{1}, y_{0} \in \overline{\operatorname{Is}(Y)}-\operatorname{Is}(Y)$, and $x_{0} \in X-\overline{\operatorname{Is}(X)}$, it follows from Lemma 5.8 that $\operatorname{inv}(\operatorname{Clop}(Z))=(1,0,1)$.

Let $X^{l}$ and $X^{r}$ be $\eta_{1}$-sets and pick an arbitrary point $x_{l} \in X^{l}$ and an arbitrary point $x_{r} \in X^{r}$. Choose an ordinal $\gamma$ and a strictly increasing sequence $\left\{x_{\alpha}^{r}\right\}_{\alpha \in \gamma}$ in $X^{r}$ such that $\left\{x_{\alpha}^{r}\right\}_{\alpha \in \gamma}$ is cofinal in the set of $x \in X^{r}$ with $x<x_{r}$. Let $\left\{x_{k}^{l}\right\}_{k \in K}$ be an enumeration of $X^{l}$. Assume that $Y$ is a normal $P$-space with $\operatorname{inv}(\operatorname{Clop}(Y))=(n, 0,1)$ for some $n \geq 1$. Then $Y_{n}$ consists of a single point by Proposition 4.2. Let $\left\{Y_{\alpha}^{r}\right\}_{\alpha \in \gamma}$ and $\left\{Y_{k}^{l}\right\}_{k \in K}$ be collections of copies of $Y$ with $y_{\alpha}^{r}$ and $y_{k}^{l}$ representing the lone elements of $\left(Y_{\alpha}^{r}\right)_{n}$ 
and $\left(Y_{k}^{l}\right)_{n}$, respectively. Let $Z^{r}$ be the pointwise gluing of $\left\{\left(Y_{\alpha}^{r}, y_{\alpha}^{r}\right)\right\}_{\alpha \in \gamma}$ and $\left(X^{r},\left\{x_{\alpha}^{r}\right\}_{\alpha \in \gamma}\right)$ over $\gamma$, and $Z^{l}$ the pointwise gluing of $\left\{\left(Y_{k}^{l}, y_{k}^{l}\right)\right\}_{k \in K}$ and $\left(X^{l},\left\{x_{k}^{l}\right\}_{k \in K}\right)$ over $K$. Both $Z^{r}$ and $Z^{l}$ are normal $P$-spaces by Lemma 5.2. Let $L=q\left(x_{l}\right) \in Z^{l}$ and $R=q\left(x_{r}\right) \in Z^{r}$. Finally, let $Z$ be the pointwise gluing of $\left(Z^{l}, L\right)$ and $\left(Z^{r}, R\right)$. We claim that $\operatorname{inv}\left(\operatorname{Clop}\left(Z^{l}\right)\right)=(n, 1,0)$ and that $\operatorname{inv}(\operatorname{Clop}(Z))=(n+1,0,1)$.

To prove that $\operatorname{inv}\left(\operatorname{Clop}\left(Z^{l}\right)\right)=(n, 1,0)$ it suffices, by Proposition 4.2, to show that $\operatorname{Is}\left(Z_{n}^{l}\right)=\emptyset$ and $Z_{n}^{l} \neq \emptyset$. The latter follows from Lemma 5.6 because $y_{k}^{l} \in\left(Y_{k}^{l}\right)_{n}$ for all $k \in K$; in particular, note that $L \in Z_{n}^{l}$ because $x_{l}=x_{k}^{l}$ for some $k \in K$ and $q\left(x_{k}^{l}\right)=q\left(y_{k}^{l}\right)$. As for the former, let $G \in \operatorname{Clop}\left(Z^{l}\right)$ with $G \cap Z_{n}^{l} \neq \emptyset$. Then $q^{-1}(G) \cap X^{l} \neq \emptyset$, for otherwise $\operatorname{gp}\left(q^{-1}(G)\right)=\emptyset$ and $G \cap Z_{n}^{l}=\emptyset$ by Lemma 5.4. Now $q^{-1}(G) \cap X^{l}$ must be infinite because it is clopen in $X^{l}$, and $X^{l}$ has no isolated points. Since $q\left(x_{k}^{l}\right)=q\left(y_{k}^{l}\right)$ for every $k \in K$, it follows that $G \cap Z_{n}^{l}$ is infinite and hence that $\operatorname{Is}\left(Z_{n}^{l}\right)=\emptyset$.

To prove that $\operatorname{inv}(\operatorname{Clop}(Z))=(n+1,0,1)$ it suffices, by Lemma 5.8 , to show that $Z_{n+1}^{l}=\emptyset=Z_{n+1}^{r}, R \in \overline{\operatorname{Is}\left(Z_{n}^{r}\right)}-\operatorname{Is}\left(Z_{n}^{r}\right)$, and $L \in Z_{n}^{l}-\overline{\operatorname{Is}\left(Z_{n}^{l}\right)}$. We have already seen that $L \in Z_{n}^{l}$ and $\operatorname{Is}\left(Z_{n}^{l}\right)=\emptyset$, from which it follows that $Z_{n+1}^{l}=\emptyset$ and $L \in Z_{n}^{l}-\overline{\operatorname{Is}\left(Z_{n}^{l}\right)}$. That $R \in \overline{\operatorname{Is}\left(Z_{n}^{r}\right)}-\operatorname{Is}\left(Z_{n}^{r}\right)$ and $Z_{n+1}^{r}=\emptyset$ both follow from Lemma 5.7.

So by induction there are, for every integer $n \geq 0$, normal $P$-spaces whose clopen algebras have the invariants $(n, 0,1)$ and also normal $P$-spaces whose clopen algebras have the invariants $(n, 1,0)$. Fix $n \geq 0$ and let $m$ be a countable cardinal greater than zero. Next we build a normal $P$-space whose clopen algebra has invariants $(n, 0, m)$. Let $\left\{Y_{k}\right\}_{k \in K}$ be a collection of normal $P$-spaces such that $|K|=m$ and $\operatorname{inv}\left(\operatorname{Clop}\left(Y_{k}\right)\right)=(n, 0,1)$ for each $k \in K$. Let

$$
X=\bigoplus_{k \in K} Y_{k}
$$

From Proposition 4.1 we know that $\left|\operatorname{Is}\left(\left(Y_{k}\right)_{n}\right)\right|=1$ and $\left(Y_{k}\right)_{n}=\overline{\operatorname{Is}\left(\left(Y_{k}\right)_{n}\right)}$ for each $k \in K$. So $\left|\operatorname{Is}\left(X_{n}\right)\right|=m$ and $X_{n}=\overline{\mathrm{Is}\left(X_{n}\right)}$ both follow from Lemma 5.5. Hence $\operatorname{inv}(\operatorname{Clop}(W))=(n, 0, m)$ by Proposition 4.2.

Next we build a normal $P$-space whose clopen algebra has invariants $(n, 1, m)$. Let $Y$ be a normal $P$-space with $\operatorname{inv}(\operatorname{Clop}(Y))=(n, 1,0), X$ be a normal $P$-space with $\operatorname{inv}(\operatorname{Clop}(X))=(n, 0, m)$, and $W=X \oplus Y$. Then $\left|\operatorname{Is}\left(X_{n}\right)\right|=m, X_{n}=\overline{\mathrm{Is}\left(X_{n}\right)}, \operatorname{Is}\left(Y_{n}\right)=\emptyset$, and $Y_{n} \neq \overline{\mathrm{Is}\left(Y_{n}\right)}$. It follows from Lemma 5.5 that $\left|\operatorname{Is}\left(W_{n}\right)\right|=m$ and $W_{n} \neq \overline{\operatorname{Is}\left(W_{n}\right)}$. Hence, by Proposition 4.2, $\operatorname{inv}(\operatorname{Clop}(W))=(n, 1, m)$ as desired.

Finally, we build a normal $P$-space whose clopen algebra has the invariants $(\omega, 0,0)$. For each integer $j \geq 0$ let $Y_{j}$ be a normal $P$-space with 
$\operatorname{inv}\left(\operatorname{Clop}\left(Y_{j}\right)\right)=(j, 0,1)$. Let

$$
W=\bigoplus_{j \geq 0} Y_{j}
$$

By Proposition 4.2, $\left(Y_{j}\right)_{n} \neq \emptyset$ whenever $n \geq 0$ and $j \geq n$. So $W_{n} \neq \emptyset$ for all $n \geq 0$ by Lemma 5.5. Thus $\operatorname{inv}(\operatorname{Clop}(X))=(\omega, 0,0)$ by Proposition 4.2 and the proof of Theorem 1.1 is complete.

\section{References}

[1] Yu. L. Ershov, Decidability of the elementary theory of relatively complemented lattices and the theory of filters, Algebra i Logika Sem. 3 (1964), no. 3, 17-38 (in Russian).

[2] L. Gillman and M. Henriksen, Concerning rings of continuous functions, Trans. Amer. Math. Soc. 77 (1954), 340-362.

[3] L. Gillman and M. Jerison, Rings of Continuous Functions, Grad. Texts in Math. 43, Springer, New York, 1976 (reprint of the 1960 edition).

[4] S. Koppelberg, Handbook of Boolean Algebras, Vol. 1, J. D. Monk and R. Bonnet (eds.), North-Holland, Amsterdam, 1989.

[5] M. H. Stone, The theory of representations for Boolean algebras, Trans. Amer. Math. Soc. 40 (1936), 37-111.

[6] A. Tarski, Arithmetical classes and types of Boolean algebras, Bull. Amer. Math. Soc. 55 (1949), 64.

[7] B. Wynne, Decidable theories of non-projectable l-groups of continuous functions, Ann. Pure Appl. Logic 146 (2007), 21-39.

Bard College at Simon's Rock

Box \#143

84 Alford Road

Great Barrington, MA 01230-1978, U.S.A.

E-mail: bwynne@simons-rock.edu

Received 19 March 200\%;

in revised form 31 July 2008 